\title{
Entropy and Existence of Universe
}

\author{
Chetan Subedi \\ Prithwi Narayan Campus, Pokhara,email: subedi_chetan@yahoo.com
}

A technical physical word which measures the lack of order that exists in a system is entropy. It is a' thermodynamic state quantity' that measures randomness or the disorder of the molecule of the system. The entropy of a substance varies directly with temperature, the lower temperature the lower entropy, the higher temperature, the higher entropy and the higher disorder. The entropy of a substance in gaseous state is more than in the liquid state, because the molecule are more free to move about in great disorder in a gas than in liquid. Moreover the entropy is more in the liquid state than in the solid state, as the molecules are freer to move in a liquid state than in the solid state. Hence when ice is converted in to water and then into steam, the entropy and disorder of molecules increases.

At absolute zero of temperature the thermal motion completely disappears, so that the disorder and hence entropy tend to zero and the molecules of a substance are in perfect order i.e. well arranged, but absolute zero of temperature is only an idealization. Notice that, if a system will reach a point where the room, the glass and the contents of the glass will be at the same temperature. In this situation nothing else can happen although thermal energy does exist in the room. From this we can conclude that if the whole world were at the same temperature one could not convert any of its heat energy into work. If we do work in room temperature slowly enough, the room temperature does not change and we can convert work into heat, but it is not possible to convert heat back into a work at a given temperature.

For example, we have a suit of playing card arranged numerically; the sequence of card is highly organized. If we throw the cards into the air and collected them we will almost surely find that they have been placed at random, when the cards are tossed there are many ways for them to be disordered, while there is only one way for them to come together again in their original sequence. Therefore the chance of disordered sequence is more probable than the ordered one. The same law of chance applies to any physical and chemical process.

It is difficult to form a physical concept of entropy as there is nothing physical to represent it and it can't be felt like temperature or pressure. Entropy of a substance remains constant in reversible process but increase in all irreversible process. Since no process is absolutely reversible, there is always least small gain in the entropy. A reversible process is only an idealization. All the physical operations in the universe are irreversible, for such operation a certain amount of energy of the universe become unavailable for useful work and is added to the universe in the form of heat through friction, conduction or radiation. In this way, in distinct future all energies existing in different forms will be converted into heat energy and will not be available for the conversion into mechanical work, i.e. available energy of the universe will tend to zero. It will correspond to a state of maximum entropy and all temperature differences between various bodies of the universe will be equalized due to convection. No heat engine will then be able to work, because no heat flow would be possible due to the uniformity of temperature throughout the universe.

Thus we reach a conclusion that at a certain point in the distant future, the whole universe will be a uniform, energy is running downhill, in which there will no available energy to do work, and this condition is known as the" heat death of universe". Locally the entropy can be lowered by external action, e.g. solar heating action, and that this applies to machines such as refrigerator where the entropy in the cold chamber is being reduced, this local decrease in entropy is however only possible at the expense of an entropy increase in the surrounding.

We therefore conclude that universe is not a fluctuation, and that the order is a memory of condition when things started. For some reason, the universe at one time had very low entropy for it's energy content, and since the entropy has increased. So that it is the way towards the future. That is the origin of all irreversibility, that is what makes the process of growth and decay, that makes us remember the past and not the future, remember the things which are closer to that moment in the history of the universe when the order was higher than now, and why we are not able remember things where the disorder is higher than now, which we call the future.

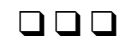

